\title{
Significance of Dungey-cycle flows in Jupiter's and Saturn's magnetospheres, and their identification on closed equatorial field lines
}

\author{
S. V. Badman and S. W. H. Cowley \\ Department of Physics \& Astronomy, University of Leicester, Leicester LE1 7RH, UK \\ Received: 29 September 2006 - Revised: 27 February 2007 - Accepted: 6 April 2007 - Published: 8 May 2007
}

\begin{abstract}
We consider the contribution of the solar winddriven Dungey-cycle to flux transport in Jupiter's and Saturn's magnetospheres, the associated voltages being based on estimates of the magnetopause reconnection rates recently derived from observations of the interplanetary medium in the vicinity of the corresponding planetary orbits. At Jupiter, the reconnection voltages are estimated to be $\sim 150 \mathrm{kV}$ during several-day weak-field rarefaction regions, increasing to $\sim 1 \mathrm{MV}$ during few-day strong-field compression regions. The corresponding values at Saturn are $\sim 25 \mathrm{kV}$ for rarefaction regions, increasing to $\sim 150 \mathrm{kV}$ for compressions. These values are compared with the voltages associated with the flows driven by planetary rotation. Estimates of the rotational flux transport in the "middle" and "outer" magnetosphere regions are shown to yield voltages of several MV and several hundred $\mathrm{kV}$ at Jupiter and Saturn respectively, thus being of the same order as the estimated peak Dungeycycle voltages. We conclude that under such circumstances the Dungey-cycle "return" flow will make a significant contribution to the flux transport in the outer magnetospheric regions. The "return" Dungey-cycle flows are then expected to form layers which are a few planetary radii wide inside the dawn and morning magnetopause. In the absence of significant cross-field plasma diffusion, these layers will be characterized by the presence of hot light ions originating from either the planetary ionosphere or the solar wind, while the inner layers associated with the Vasyliunas-cycle and middle magnetosphere transport will be dominated by hot heavy ions originating from internal moon/ring plasma sources. The temperature of these ions is estimated to be of the order of a few $\mathrm{keV}$ at Saturn and a few tens of $\mathrm{keV}$ at Jupiter, in both layers.
\end{abstract}

Keywords. Magnetospheric physics (Magnetospheric configuration and dynamics; Planetary magnetospheres; Plasma convection; Solar wind-magnetosphere interactions)

Correspondence to: S. V. Badman

(svb4@ion.le.ac.uk)

\section{Introduction}

Experimental investigation of the large-scale structure and dynamics of the magnetospheres of Jupiter and Saturn have become possible in recent years through data obtained both by spacecraft in situ and by remote-sensing instruments. The Galileo orbiter mission has been the principal source of in situ data at Jupiter (e.g. Krupp et al., 2001; Frank et al., 2002; Kronberg et al., 2005; Waldrop et al., 2005), as well as providing remote sensing observations using radio emissions (e.g. Louarn et al., 2000). A similar body of information is currently being gathered at Saturn by the Cassini orbiter (e.g. Bunce et al., 2005; Dougherty et al., 2005; Gurnett et al., 2005; Young et al., 2005), now also including energetic neutral atom imaging of magnetospheric dynamics (e.g. Krimigis et al., 2005; Paranicas et al., 2005). Information on global dynamics can also be obtained from studies of polar UV auroras observed by the Hubble Space Telescope (HST), at both Jupiter (e.g. Grodent et al., 2003a, b; Nichols et al., 2007) and Saturn (e.g. Gérard et al., 2004; Clarke et al., 2005; Grodent et al., 2005; Badman et al., 2005). The observed auroral emissions relate both to precipitation from hot plasma populations produced within the magnetosphere, and to intense structured field-aligned current systems that are associated with magnetosphere-ionosphere momentum transfer (Cowley et al., 2004a, b, 2005a, b; Gustin et al., 2004, 2006; Nichols and Cowley, 2004; Jackman and Cowley, 2006).

From a theoretical viewpoint, two major aspects of largescale dynamics must be considered, together with their mutual interaction. The first is the dynamics of the flow driven by the rotation of the planet through ion-neutral collisions in the ionosphere, combined with internal sources of plasma deriving from moon atmospheres and surfaces, and from ring grains. Such plasma is picked up by the rotational flow in the inner regions of the magnetosphere, transported outward by centrifugal action, and then lost by some process acting in the outer magnetosphere (Hill, 1979; Siscoe and Summers, 1981; Hill et al., 1983; Southwood and Kivelson, 1989;

Published by Copernicus GmbH on behalf of the European Geosciences Union. 


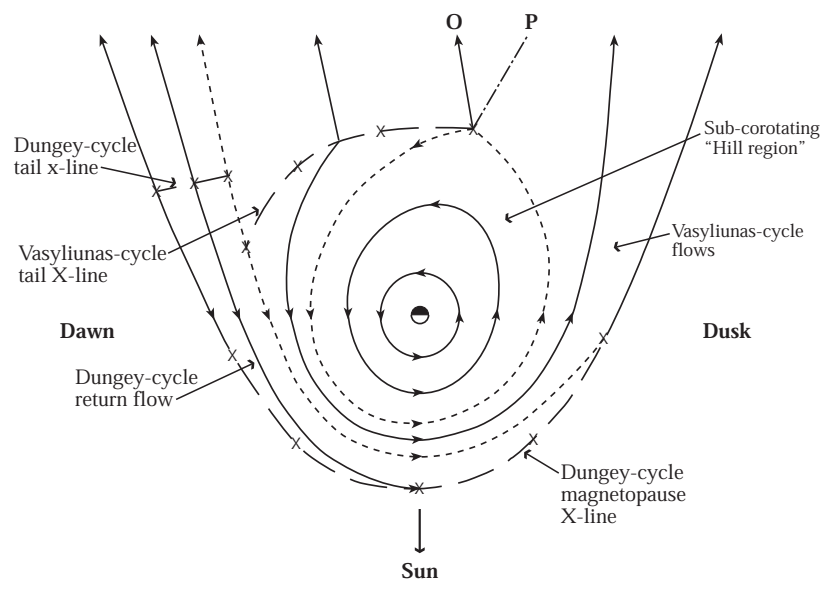

Fig. 1. Sketch of the flows in the jovian equatorial plane, with the direction toward the Sun at the bottom of the figure, dawn to the left, and dusk to the right. Solid lines with arrows show plasma streamlines, while dashed lines with arrows show streamlines which separate flow regions with differing origins and characteristics as indicated. Dashed lines with "X"s indicate X-type reconnection lines, while the solid line marked "O" indicates the O-type line of the Vasyliunas-cycle plasmoid which is ejected down-tail (which is a streamline). The dot-dashed line marked " $\mathrm{P}$ " is the outer boundary of the plasmoid, which asymptotes to the dusk tail magnetopause (from Cowley et al., 2003b).

Pontius, 1997; Bespalov et al., 2006). Vasyliunas (1983) suggested that mass-loaded flux tubes in the outer corotating region will generally be radially restrained on the dayside by the solar wind dynamic pressure, but may then stretch out down-tail as they rotate into the dusk sector, eventually pinching off to form a tailward-propagating plasmoid. The mass-reduced closed portions of these flux tubes then return to the dayside via dawn, where they again become massloaded by radial diffusion from the inner regions and stretch out once more as they rotate into the dusk sector. Here we refer to this process as the Vasyliunas-cycle. The second aspect of magnetospheric dynamics is the cyclical flow driven by the interaction between the magnetosphere and the solar wind. Here we assume from evidence at Earth that the interaction is mediated principally by magnetic reconnection occuring at the magnetopause, producing open flux tubes that are transported by the solar wind flow to the tail (e.g. Cowley et al., 2003a). The flux then returns sunward to the dayside following reconnection episodes in the tail to complete the transport cycle. This process was first described for the terrestrial magnetosphere by Dungey (1961), and is referred to here as the Dungey-cycle. In this note we discuss the relative magnitudes of the flux transport by rotational and Dungeycycle dynamics in Jupiter's and Saturn's magnetosphere, and indicate how the contribution of the Dungey-cycle may potentially be identified in equatorial flows. In the next section we discuss the nature of the overall flow system before es- timating the contributions from the rotational and Dungeycycle flows in following sections.

\section{Equatorial flows}

Conceptual models of the large-scale magnetospheric field and flow containing both rotational/Vasyliunas-cycle and Dungey-cycle systems have been discussed for Jupiter by Cowley et al. (1996, 2003b), and for Saturn by Cowley et al. (2004a). A central purpose of these models was to show how the two flow systems can co-exist on a continuous basis. The equatorial flow in the jovian model discussed by Cowley et al. (2003b) is sketched in Fig. 1, and forms the basis of the discussion given here; the flow in the Saturn model is essentially similar. In Fig. 1 we look down onto the magnetospheric equatorial plane from the north, with the direction towards the Sun at the bottom of the diagram, dawn to the left, and dusk to the right. The solid lines with arrows show plasma streamlines, with three regimes of flow being depicted following the above discussion, which are separated by the dashed-line streamlines. The inner region is dominated by the production and pick-up of plasma from internal sources (principally Io at Jupiter) by the rotating flow, followed by its outward radial transport through small-scale "diffusive" motions not depicted in the figure. The flow is near-rigidly corotating with the planet in the innermost region, but as a consequence of outward transport, increasingly departs towards lower angular velocities at increasing distances (e.g. Hill, 1979; Pontius, 1997; Cowley et al., 2002). This region is then surrounded by a flow layer where the flux tubes, still driven by planetary rotation, take part in the Vasyliunas-cycle. Here the closed mass-loaded flux tubes flow radially outward into the dusk tail before pinching-off to form a tailward-flowing plasmoid, as indicated above. The associated reconnection line and inner boundary of the plasmoid is indicated in the figure by the dashed line with "X"s, marked "Vasyliunas-cycle tail X-line". The central O-type neutral line of the plasmoid is indicated by the line marked "O" (which is also a streamline), while the outer boundary of the plasmoid is indicated by the dot-dashed line marked " $\mathrm{P}$ " which eventually asymptotes to the dusk magnetopause.

The solar wind-generated flow in the figure, associated with the Dungey-cycle, is then confined to the dawn side of the system, where the "Dungey-cycle tail X-line" is located. Open tail flux tubes which are closed at this reconnection site flow sunward to the dayside magnetopause in a layer adjacent to the dawn magnetopause, where (in the steady state) they become open once more due to reconnection with the magnetosheath field. After this the open tubes become transported poleward out of the plane of the diagram, and then into the tail lobes due to the anti-sunward flow of the solar wind. Open flux tubes are then removed from the lobes at the reconnection site on the dawn side of the tail to complete the cycle. Flux tubes disconnected from the planet 
at this site flow tailward on the dawn side of the tailwardpropagating plasmoid. Overall, this picture shows that these large-scale plasma flow systems can co-exist on a continuous basis. It is nevertheless recognised that in reality both Vasyliunas-cycle and Dungey-cycle processes are likely to be time-dependent (e.g. Woch et al. 2004; Cowley et al., 2005a), and may also involve nightside flows that are more spatially structured than those depicted here, as speculated upon by Kivelson and Southwood (2005).

In terms of the observed plasma regions in Jupiter's magnetosphere, we suppose that the mass-loaded sub-corotating region corresponds to the jovian "middle magnetosphere", which is dominated by the presence of an equatorial plasmacurrent sheet containing outwardly-diffusing cool iogenic plasma. We also suppose that the surrounding region of mass-reduced flux tubes flowing around the boundary via dawn from the Vasyliunas- and Dungey-cycle reconnection sites in the tail together form the "outer magnetosphere" region on the dayside, characterised by relatively strong southward-pointing fields in the equatorial plane and the lack of a central current sheet, as originally suggested by Cowley et al. (1996). In subsequent discussions Cowley et al. (2003b, 2005b) emphasised the Dungey-cycle contributions to this layer, while Kivelson and Southwood (2005) emphasise the Vasyliunas-cycle contribution. Here we will discuss in more detail the relative contributions of these two processes to the formation of the layer. We also recognise that the character of the layer will evolve as the plasma is transported from dawn around the dayside, due to reconnection-associated flux erosion at the magnetopause as depicted in Fig. 1, as well as to radial transport of plasma from the interior region (Kivelson and Southwood, 2005).

\section{Rotational and Dungey-cycle flux transport at Jupiter and Saturn}

It is evident from the above discussion that the flux transport driven by planetary rotation and by the Dungey-cycle will dominate in differing parts of the magnetosphere. Generally, rotation-driven flows will dominate in the inner regions of closed field lines, while the Dungey-cycle is critical in the formation of open tail lobes, which, at Jupiter, extend antisunward of the planet by several AU to roughly the orbit of Saturn (Behannon et al., 1983; Cowley et al., 2003b). It is nevertheless important for an overall understanding of the nature of these systems to compare quantitatively the flux transport due to these processes, such that we can assess, for example, the contribution of the Dungey-cycle return flows to the equatorial transport in the outer magnetosphere. These topics are conveniently discussed in terms of the relative voltages associated with the motional electric field $\boldsymbol{E}=-\boldsymbol{v} \times \boldsymbol{B}$ due to these flow systems, where $\boldsymbol{v}$ is the plasma velocity and $\boldsymbol{B}$ the magnetic field, since by Faraday's law a voltage of $1 \mathrm{~V}$ corresponds to a flux transport of $1 \mathrm{Wbs}^{-1}$. In this section we thus first review recent results concerning the Dungeycycle voltage at Jupiter and Saturn, and then compare them with estimates of the flow driven by planetary rotation.

\subsection{Flux transport associated with Dungey-cycle flow}

Until recent times only rather crude spot estimates had been made of the magnetopause reconnection rates that initiate the Dungey-cycle at Jupiter and Saturn (e.g. Kennel and Coroniti, 1975). Recently, however, more detailed estimates have been published, based on the growing collection of interplanetary data obtained in the vicinity of the planetary orbits by the Ulysses and Cassini spacecraft (Jackman et al., 2004; Nichols et al., 2006). These studies employed an empirical formula for the magnetopause reconnection voltage in terms of upstream interplanetary parameters, which was validated and adapted from studies at the Earth (e.g. Perrault and Akasofu, 1978; Milan et al., 2004), given by

$V_{D C}=v_{S W} B_{\perp} L_{0} \cos ^{4}(\theta / 2)$

Here $v_{S W}$ is the velocity of the solar wind, $B_{\perp}$ is the magnitude of the interplanetary magnetic field (IMF) vector perpendicular to the flow, and $\theta$ is the "clock angle" of this vector measured from the planet's north magnetic axis projected onto a plane perpendicular to the planet-Sun line. Length $L_{0}$ is then such that the field-perpendicular width of the solar wind channel which reconnects with the planetary field is given by $L_{0} \cos ^{4}(\theta / 2)$. This width is a maximum equal to $L_{0}$ when $\theta=0^{\circ}$ i.e. when the IMF points northward opposite to the equatorial planetary field, and falls to small values beyond $\theta \sim 90^{\circ}$. From a study of reconnection rates at Earth, Milan et al. (2004) found a value for the scale length $L_{0} \sim 5 R_{E}$ i.e. approximately half the sub-solar magnetopause stand-off distance. For Jupiter and Saturn $L_{0}$ has therefore been scaled according to the size of the magnetospheres, such that $L_{0} \sim 30 R_{J}$ (on average) at Jupiter, while $L_{0} \sim 10 R_{S}$ at Saturn.

The interplanetary medium at the orbital distances of Jupiter and Saturn typically exhibits strong variations during each $\sim 25$-day solar rotation due to the effect of both corotating interaction regions (CIRs) and coronal mass ejection (CME) events. As a consequence, the reconnection voltages estimated from Eq. (1) show corresponding strong temporal variations over such intervals due principally to changes in the strength of the IMF, upon which further modulation is superposed at shorter periods due to the clock angle effect. Lowest field strengths and voltages occur during several-day CIR rarefaction regions during which the solar wind speed falls with time, while highest field strengths and voltages occur during few-day CIR compression regions when the solar wind speed increases (usually across paired shocks) and during few-day CMEs. Considering first the results for Jupiter, Nichols et al. (2006) studied three extended intervals of Ulysses data as the spacecraft flew near Jupiter's orbital path during 1992, 1998 and 2004, and one further interval 
of data obtained by Cassini during its fly-by of Jupiter at the end of 2000. The data thus span a complete solar cycle, though the variations over the cycle were found to be relatively modest. Typical magnetopause reconnection voltages were estimated to be $V_{D C} \sim 150 \mathrm{kV}$ during rarefaction regions, which occur $\sim 90 \%$ of the time, increasing by an order of magnitude to $V_{D C} \sim 1 \mathrm{MV}$ during CIR compressions and CMEs, which occur $\sim 10 \%$ of the time (i.e. in one or two intervals of $\sim 2-3$ days total duration during each solar rotation). These and subsequent voltage values are listed in Table 1, for purposes of comparison. The open flux produced during the extended rarefaction intervals integrates to $\sim 300 \mathrm{GWb}$ over one solar rotation, while that produced in the relatively short-lived compression regions integrates to $\sim 200 \mathrm{GWb}$. The total open flux produced over a solar rotation is thus $\sim 500 \mathrm{GWb}$, corresponding to an overall average magnetopause reconnection rate of $\sim 250 \mathrm{kV}$. Since the jovian magnetotail contains $\sim 300-500 \mathrm{GWb}$ of flux per lobe, the averaged tail flux replenishment time is thus estimated to be $\sim 15-25$ days, with a consequent tail length of $\sim 4-6 \mathrm{AU}$ consistent with the above discussion.

Considering now the case for Saturn, Jackman et al. (2004) employed a $\sim 6$-month interval of interplanetary data measured by Cassini as it approached Saturn at the start of 2004, corresponding to the declining phase of the solar cycle. Their results (again listed in Table 1) indicate typical reconnection voltages of $V_{D C} \sim 25 \mathrm{kV}$ during rarefaction regions, which occur $\sim 85 \%$ of the time, increasing to $V_{D C} \sim 150 \mathrm{kV}$ during CIR compressions, which occur $\sim 15 \%$ of the time (i.e. one or two intervals of $\sim 4$ days total duration during each solar rotation). The open flux produced during rarefaction regions then integrates to $\sim 50 \mathrm{GWb}$ in each solar rotation, while that produced in compression regions also integrates to $\sim 50 \mathrm{GWb}$. The total amount of open flux created is then $\sim 100 \mathrm{GWb}$ per solar rotation, corresponding to an averaged magnetopause reconnection rate of $\sim 45 \mathrm{kV}$. Since the kronian magnetotail contains $\sim 25-40 \mathrm{GWb}$ of open flux per lobe, the averaged tail flux replenishment time is estimated to be $\sim 6-10$ days, with a consequent tail length of $\sim 1.5-$ $2.5 \mathrm{AU}$.

It should be noted here that the values determined above using Eq. (1) are estimates of the reconnection rate at the magnetopause, while we are particularly interested in the Dungey-cycle contribution to flux transport on closed field lines in the outer magnetosphere following reconnection in the tail, as depicted in Fig. 1. It is firstly evident that the averaged Dungey-cycle tail reconnection rate, and consequent closed flux transport rate in the outer magnetosphere, must be equal to the averaged magnetopause reconnection rate, estimated above to be $\sim 250 \mathrm{kV}$ at Jupiter and $\sim 45 \mathrm{kV}$ at Saturn. Beyond this it seems reasonable to assume that the tail reconnection rate approximately follows the variations in the magnetopause reconnection rate during each solar rotation, such that the averaged tail reconnection rate at Jupiter would be $\sim 150 \mathrm{kV}$ during rarefaction regions, increasing to $\sim 1 \mathrm{MV}$ during CIR compression regions and CMEs. The corresponding values for Saturn are $\sim 25 \mathrm{kV}$ for rarefaction regions and $\sim 150 \mathrm{kV}$ for compressions, in good agreement with the findings of Badman et al. (2005) based on analysis of auroral data from the January 2004 Cassini-HST campaign. In addition to this, however, we note that at Earth tail reconnection tends to be impulsive, resulting in substorms and other nightside flow burst phenomena that may be triggered internally or by interplanetary events (e.g. Lyons et al., 1997; Boudouridis et al., 2003, 2004; Milan et al., 2006). The consequence is that while the magnetopause reconnection rate at Earth, averaged over all interplanetary conditions, is $\sim 30 \mathrm{kV}$, the typical tail reconnection rate, when it occurs, is $\sim 90 \mathrm{kV}$, lasting for about one third of the time (Milan et al., 2004, 2006). It should therefore be recognised that the typical tail reconnection rates at Jupiter and Saturn, when they do occur, may be higher than the magnetopause reconnection rates estimated above, but lasting for correspondingly shorter intervals. If a similar factor of three applies at Jupiter as at Earth, for example, the tail reconnection rate might be $\sim 3 \mathrm{MV}$ during compression regions, though occurring for a total of only $\sim 1$ day during each solar rotation. Similarly, the tail reconnection rate at Saturn might be $\sim 450 \mathrm{kV}$, occurring for a total of $\sim 1.5$ days per solar rotation according to the above discussion.

3.2 Flux transport associated with rotational flow and comparison with the Dungey-cycle

We now consider the flux transport driven by the planetary rotation and compare it with that due to the Dungey-cycle flows considered above. If planetary magnetic flux $\Phi$ rotates at angular velocity $\omega$, such that the rotation period is $T=2 \pi / \omega$, then the rotational flux transport and voltage associated with the flow is given by

$V_{\mathrm{ROT}}=\frac{\Phi}{T}=\frac{\Phi \omega}{2 \pi}$.

Generally the plasma will sub-corotate at a fraction $k$ of the planet's angular velocity $\Omega_{P}$, such that $\omega=k \Omega_{P}$, where for sub-corotation $0<k<1$. We then have

$V_{\mathrm{ROT}}=\frac{k \Phi \Omega_{P}}{2 \pi}=\frac{k \Phi}{T_{P}}$,

where $T_{P}=2 \pi / \Omega_{P}$ is the planetary rotation period. Previous simple estimates of the rotational voltage have generally considered the whole of the planetary flux over a given hemisphere and have assumed rigid corotation $(k=1)$, as will initially be assumed here. To estimate the amount of flux in one hemisphere we integrate the principal dipole component of the internal planetary field over the hemisphere. For Jupiter we use the VIP-4 jovian magnetic field model derived by Connerney et al. (1998) to estimate the total hemispheric magnetic flux to be $\sim 14000 \mathrm{GWb}$, such that for rigid corotation with a $\sim 9.9 \mathrm{~h}$ rotation period, the associated flux 
Table 1. Summary of estimated Dungey-cycle and rotational voltages for Jupiter and Saturn.

\begin{tabular}{lll}
\hline Voltage estimates & Jupiter (MV) & Saturn (MV) \\
\hline Rotational voltage (total) & 400 & 12 \\
Rotational voltage (middle magnetosphere or ring current region) & 2.5 & $0.4-0.6$ \\
Rotational voltage (outer magnetosphere) & $2-4$ & 0.18 \\
Dungey-cycle voltage (compression region) & 1 & 0.15 \\
Dungey-cycle voltage (rarefaction region) & 0.15 & 0.025 \\
Dungey-cycle voltage (average) & 0.25 & 0.045 \\
\hline
\end{tabular}

transport is $V_{\text {ROT }} \sim 400 \mathrm{MV}$ (Table 1). Similarly, using the Cassini model of Saturn's magnetic field (Dougherty et al., 2005), we find that at Saturn the total hemispheric magnetic flux is $\sim 480 \mathrm{GWb}$, so that with a $\sim 10.8 \mathrm{~h}$ rotation period we obtain $V_{\text {ROT }} \sim 12 \mathrm{MV}$ (Table 1 ). It may be thought that these voltage values represent overestimates since the plasma sub-corotates throughout much of the outer magnetosphere. However, this sub-corotation region maps to within $\sim 20^{\circ}$ of the magnetic pole at Jupiter and within $\sim 25^{\circ}$ of the pole at Saturn, corresponding to only $\sim 10 \%$ and $20 \%$, respectively, of the total planetary magnetic flux (Cowley et al., 2004b, 2005b), as will be discussed further below. If the plasma rotates on average at approximately half the planetary angular velocity throughout this region, then the above rotational voltage estimates will be reduced by only $\sim 5 \%$ and $10 \%$, respectively, due to this effect. These considerations thus produce only minor corrections to the above total rotational voltage values.

It is thus evident that these rotational voltages very greatly exceed the averaged Dungey cycle flux transports discussed above. Even the estimated compression region Dungey-cycle voltages at Jupiter and Saturn are only $\sim 1 \%$ of these values, or less, which may be taken to imply that the Dungeycycle flows are quite negligible at these planets, except for the production of the extended magnetotail. By comparison, the rotational voltage at Earth computed on the same basis is $V_{\mathrm{ROT}} \sim 90 \mathrm{kV}$. The average $30 \mathrm{kV}$ Dungey-cycle reconnection rate in this case is already $30 \%$ of the rotational voltage, while the typical rate of tail reconnection, when it occurs, is comparable or larger. We therefore see that the magnetospheres of Jupiter and Saturn are very similar to each other in this respect, but are very different to the magnetosphere of the Earth.

This conclusion on the negligible role of the Dungey-cycle flows at Jupiter and Saturn considerably overstates the case, however, since most of the rotating flux considered above closes in the quasi-dipolar inner regions of the magnetosphere where the fields are strongest. As indicated above, the flux circulating in the outer parts of these magnetospheres is generally a small fraction of the total, and also usually significantly sub-corotates relative to the planet, leading to greatly reduced rotational voltage values for these regions. It may still be the case, therefore, that the Dungey-cycle contributes significantly to the flux transport in the outer regions of these magnetospheres as shown in Fig. 1. To investigate this issue we will now make some estimates of the rotational flux transport specifically in the outer regions of Jupiter's and Saturn's magnetospheres, considering both the middle magnetosphere or "ring current" regions where strong azimuthal currents are present, and the "outer magnetosphere" layer adjacent to the magnetopause where the Vasyliunas-cycle and the Dungeycycle return flow takes place, according to Fig. 1 . We begin with Jupiter and first consider the rotational flow in the middle magnetosphere, which we take to extend across the region from where corotation breaks down at $\sim 20 R_{J}$ radial distance to the outer edge at $\sim 60 R_{J}$. Using the Khurana and Kivelson (1993) model of the equatorial magnetic field, the amount of magnetic flux threading this region is estimated to be $\sim 160 \mathrm{GWb}$. The typical plasma angular velocity in this region based on Voyager and Galileo spacecraft data corresponds roughly to $k=0.6$ (e.g. Kane et al., 1995; Krupp et al., 2001) such that from Eq. (3) we obtain an estimate of the rotational voltage in Jupiter's middle magnetosphere (subscript "MM") of $V_{\text {ROT MM }} \sim 2.5 \mathrm{MV}$ (Table 1 ). This value is also in agreement with the theoretical model calculation by Nichols and Cowley (2005), which yields $V_{\text {ROT MM }} \sim 3 \mathrm{MV}$ across the same region. We can also estimate the rotational voltage across Jupiter's outer magnetosphere layer, which extends from the outer edge of the middle magnetosphere to the magnetopause in the dayside sector of the magnetosphere (see Fig. 1). Since this flux does not complete a full rotation of the planet, such that Eq. (3) is not evidently appropriate, we instead estimate the voltage by integrating the motional electric field $E_{O M}$ across the outer magnetosphere layer to obtain

$V_{\mathrm{ROT} \mathrm{OM}} \approx E_{O M} L_{O M} \approx v_{O M} B_{O M} L_{O M}$,

where $v_{O M}$ is the velocity of the plasma, $B_{O M}$ is the outer magnetosphere field strength, and $L_{O M}$ is the width of the layer. Spacecraft observations show that the outer magnetosphere layer is $\sim 15 R_{J}$ wide adjacent to the dayside magnetopause, with a field strength of $\sim 10 \mathrm{nT}$ (e.g. Acuña et al., 1983). The velocity of the plasma in this region is typically $v_{O M} \sim 200-400 \mathrm{~km} \mathrm{~s}^{-1}$ (i.e. $k \sim 0.25-0.5$ ), possibly 
depending on the state of expansion of the magnetosphere (e.g. Kane et al., 1995; Krupp et al., 2001). From Eq. (4) we then find the voltage associated with the rotational flow in the outer magnetosphere is $V_{\mathrm{ROT} \mathrm{OM}} \sim 2-4 \mathrm{MV}$ (Table 1). Comparing the voltage values in Table 1 it is thus evident that rarefaction region Dungey-cycle voltages of $V_{D C} \sim 150 \mathrm{kV}$ are still small compared with these rotational voltages in both the middle and outer regions of the magnetosphere. However, compression region Dungey-cycle voltages of $V_{D C} \sim 1 \mathrm{MV}$, although occurring only $\sim 10 \%$ of the time, are directly comparable in magnitude with those of the rotational flow in both the middle and outer magnetosphere. When such voltages occur, possibly further augmented by the impulsive tail behaviour discussed above, the Dungey-cycle flows will contribute significantly to the flow in the outer regions of Jupiter's magnetosphere.

Similarly for Saturn's magnetosphere, we first examine the rotational flows in the ring current region, analogous to the middle magnetosphere at Jupiter. Pioneer-11 and Voyager observations show that this extends from an inner edge at $\sim 6-8 R_{S}$ out to distances of $\sim 16 R_{S}$ (Connerney et al., 1983; Bunce and Cowley, 2003; Cowley and Bunce, 2003; Cowley et al., 2004b). Cowley et al. (2004b) used a magnetic field model based on Voyager data to show that the amount of magnetic flux threading the equatorial plane between these radial limits is $\sim 25-40 \mathrm{GWb}$. Voyager data show that the typical plasma angular velocity in this region corresponds to $k \sim 0.6$ (Richardson, 1986; Richardson and Sittler, 1990). From Eq. (3) we then find that the voltage associated with the rotational flow in Saturn's ring current region is $V_{\mathrm{ROT} R C} \sim 400-600 \mathrm{kV}$ (Table 1). For the outer magnetosphere, the layer between the outer edge of the ring current region and the magnetopause, we again use Eq. (4) with a width $L_{O M} \sim 6 R_{S}$ (e.g. Arridge et al., 2006), a field strength $B_{O M} \sim 5 \mathrm{nT}$ (e.g. Dougherty et al., 2005), and a flow speed of $v \sim 100 \mathrm{~km} \mathrm{~s}^{-1}$ corresponding to $k \sim 0.7$ (Richardson, 1986; Richardson and Sittler, 1990; Szego et al., 2005; Hartle et al., 2006). Equation (4) then yields an outer magnetosphere rotational voltage of $V_{\mathrm{ROT} \mathrm{OM}} \sim 180 \mathrm{kV}$ (Table 1). Comparing these values with those for the Dungey-cycle at Saturn given in Table 1 shows that, as at Jupiter, these rotational voltages are much larger than the rarefaction region Dungeycycle voltages, estimated to be $V_{D C} \sim 25 \mathrm{kV}$. However, the compression-region Dungey-cycle voltages of $\sim 150 \mathrm{kV}$ are again competitive, especially if augmented by impulsive tail behaviour, such that when these peak voltages are occurring, the Dungey-cycle flow will have a significant influence in the outer magnetosphere.

To take the argument a step further, we can compute the radial width of the layer that the Dungey-cycle "return" flow contributes to the outer magnetosphere region adjacent to the dawn magnetopause, as depicted in Fig. 1. Following Eq. (4), the voltage across the layer is $V_{D C} \approx E_{O M} L_{D C}$ where $E_{O M}$ is the electric field associated with the flow, and $L_{D C}$ is the layer width perpendicular to the magnetopause. If the plasma flow velocity in the layer is $v_{O M}$, and the magnetic field strength adjacent to the magnetopause is $B_{O M}$, as before, this becomes $V_{D C} \approx v_{O M} B_{O M} L_{D C}$, such that

$$
L_{D C} \approx \frac{V_{D C}}{v_{O M} B_{O M}} \text {. }
$$

Writing the flow velocity in terms of the planetary angular velocity, we have $v_{O M}=k R_{M P} \Omega_{P}=\frac{2 \pi k R_{M P}}{T_{P}}$, where $R_{M P}$ is the magnetopause radius. Then Eq. (5) becomes

$L_{D C} \approx \frac{V_{D C} T_{P}}{2 \pi k R_{M P} B_{O M}}$.

For rarefaction region conditions at Jupiter we use $R_{M P} \sim 90 R_{J}$ (Joy et al., 2002) in the pre-noon hours (e.g. $\sim 09: 00 \mathrm{LT}$ ), $k \sim 0.25, B_{O M} \sim 5 \mathrm{nT}$, and $V_{D C} \sim 150 \mathrm{kV}$ (Table 1) to find $L_{D C} \sim 1 R_{J}$, thus representing a minor contribution under these conditions. During CIR- or CMErelated solar wind compressions of the magnetosphere, when $R_{M P} \sim 60 R_{J}, k \sim 0.5, B_{O M} \sim 10 \mathrm{nT}$, and $V_{D C} \sim 1 \mathrm{MV}$, however, we find $L_{D C} \sim 4 R_{J}$, possibly augmented further if the tail reconnection is impulsive. This represents a more significant contribution to an outer magnetosphere layer of 10 $15 R_{J}$ thickness under these conditions. Similarly, under rarefaction region conditions at Saturn we use $R_{M P} \sim 30 R_{S}$ in the pre-noon hours (Arridge et al., 2006), $k \sim 0.7$ (Richardson, 1986; Richardson and Sittler, 1990; Szego et al., 2005; Hartle et al., 2006), and $B_{O M} \sim 4 \mathrm{nT}$ (Dougherty et al., 2005), to obtain a layer width $L_{D C} \sim 0.5 R_{S}$ when $V_{D C} \sim 25 \mathrm{kV}$. Under compression region Dungey-cycle conditions when $V_{D C} \sim 150 \mathrm{kV}$ we use $R_{M P} \sim 20 R_{S}, k \sim 0.7$, and $B_{O M} \sim 10 \mathrm{nT}$ to obtain $L_{D C} \sim 2 R_{S}$, which again may be augmented if tail reconnection is impulsive. As at Jupiter, the layer width under rarefaction region conditions at Saturn is small and essentially negligible, but under compression region conditions it becomes a significant contributor to the outer magnetosphere. We note that similar hot plasma layer widths adjacent to Saturn's magnetopause have been estimated on the basis of auroral distributions by Badman et al. (2006).

\section{Identification of Dungey-cycle flows in the equatorial magnetosphere}

The above discussion indicates that under conditions of peak Dungey-cycle tail reconnection rates associated with interplanetary CIR compressions or CMEs, equatorial "return" flow layers of non-negligible width should exist adjacent to at least the pre-noon magnetopauses at Jupiter and Saturn. The layer may become thinned in the post-noon hours, however, if significant reconnection-related flux tube erosion is present at the dayside magnetopause, as indicated in the steady-state picture shown in Fig. 1. It is then of interest to consider how such layers may be identified, relative to outer magnetosphere flow layers associated with rotational dynamics and the Vasyliunas-cycle. Although not mentioned 
in the works cited above in which this model has previously been discussed (Cowley et al., 2003b; 2004a, b), a key difference exists between these layers which could in principle allow their experimental identification. This concerns the differing sources of the hot plasma within these layers, which should result in a strongly different hot ion composition. Since the sub-corotational middle magnetosphere region and Vasyliunas-cycle flows are driven specifically by internal sources combined with planetary rotation, the ion component of these plasmas will be determined by the nature of the internal sources gases. At Jupiter, therefore, this internal plasma will be dominated by sulphur and oxygen ions originating from Io (e.g. Bagenal, 1994; Radioti et al., 2005), while at Saturn it will be dominated by oxygen and hydrogen originating from the icy moons (e.g. Young et al., 2005). The hot plasma in the Dungey-cycle "return" region, however, results from the heating of tail lobe plasma downstream from the tail reconnection site, and will thus consist principally of light ions, hydrogen and helium, originating either from the planet's ionosphere via the polar wind (potentially containing singly-charged helium), or from the solar wind (containing doubly-charged helium).

We can also estimate the temperature of the heated ions within the outer magnetosphere layer. When reconnection occurs in the tail current sheet, either on closed field lines during the Vasyliunas-cycle, or on open field lines during the Dungey-cycle, the newly-closed reconnected field lines contract towards the planet. The ions on the contracting field lines are accelerated approximately to the Alfvén speed outside the current sheet, $V_{A}=B / \sqrt{\mu_{0} m_{i} n_{i}}$, where $B$ is the field strength outside the current sheet, and $m_{i}$ and $n_{i}$ are the ion mass and number density respectively (e.g. Cowley, 1984). If we assume that the plasma is composed of a single ion species (the dominant contributor to the mass density) then the energy of these ions following acceleration will be

$$
W_{i} \approx \frac{1}{2} m_{i} V_{A}^{2} \approx \frac{1}{2} \frac{B^{2}}{\mu_{0} n_{i}} .
$$

The energy per ion thus depends only on the field strength and number density, and not on the ion mass. Considering first the jovian tail current sheet at radial distances of $\sim 100 R_{J}$ where $B \sim 6 \mathrm{nT}$ and $n_{i} \sim 0.01 \mathrm{~cm}^{-3}$ (e.g. Russell et al., 1998; Frank et al., 2002; Kronberg et al., 2005), we obtain from Eq. (7) an ion energy of $\sim 10 \mathrm{keV}$. This value is in agreement with ion temperatures in the current sheet presented by Frank et al. (2002). As the plasma is transported from the tail towards the dayside, its energy may be augmented by factors of $\sim 2-3$ due to adiabatic compression of the contracting flux tubes, such that we then expect the production of a hot-ion plasma with temperatures of a few tens of $\mathrm{keV}$ in the dayside outer magnetosphere, both in the Dungey-cycle and the Vasyliunas-cycle layers. Considering now Saturn's magnetotail at a distance of a few tens of $R_{S}$ from the planet, we take values of $B \sim 3 \mathrm{nT}$ and $n_{i} \sim 0.01 \mathrm{~cm}^{-3}$ (Ness et al., 1981; Richardson, 1986) to obtain using Eq. (7) an ion energy of $\sim 2 \mathrm{keV}$. Again, this energy may be augmented by factors of $\sim 2-3$ due to adiabatic compression of the plasma as it rotates round to the dayside magnetosphere, such that we anticipate hot ion layers of $\sim 5 \mathrm{keV}$ energy in the dayside outer magnetosphere in this case.

Thus to the extent that cross-field diffusion does not "blur" the ion composition, it should be possible to identify Dungey-cycle "return" flows in the outer magnetospheres of Jupiter and Saturn, and to distinguish them from regions dominated by the dynamics associated with the transport of plasma from internal sources, by examination of the dominant ion species at energies from a few $\mathrm{keV}$ to a few tens of keV. According to Fig. 1, the Dungey-cycle flows should form a hot-plasma layer adjacent to the magnetopause in the pre-noon hours if magnetopause reconnection is on-going, but will be more broadly distributed across the dayside region including the dusk sector if it is not. Similar comments apply to the layers of antisunward flow tailward of the reconnection sites in the centre plane of the tail. The tailwardflowing plasma sheet associated with the Dungey-cycle and the closure of open lobe flux on the dawn flank will consist primarily of light ions with $\sim \mathrm{keV}$ energies, while that associated with the tailward-propagating plasmoid formed by the Vasyliunas-cycle will consist principally of heavy ions with similar energies. As indicated above, however, Kivelson and Southwood (2005) have recently speculated that the nightside outflow in Jupiter's magnetotail is associated with sequential outflow events that involve episodes of plasmoid ejection and open flux closure on much smaller spatial scales than is envisaged in Fig. 1. In this case the Vasyliunas-cycle and Dungey-cycle "return" flows may become inextricably interlinked, such that no separate flow components with individual ion composition signatures may occur.

With regard to observations, we note that no directly relevant data concerning the composition of ions at energies of a few $\mathrm{keV}$ to a few tens of $\mathrm{keV}$ per ion have yet been published for the outer magnetosphere regions of either Jupiter or Saturn. This paper therefore sets out the groundwork for future experimental tests of the ideas on magnetospheric structure and dynamics at these giant planets, outlined in Fig. 1. Unfortunately it appears unlikely that definitive information can be derived for Jupiter's outer magnetosphere from existing data from the Galileo orbiter. The reader is referred to publications by e.g. Frank et al. (2002) and Frank and Paterson (2004) for discussion of the limitations in ion mass resolution of the Galileo instrumentation in the above energy range. Mass-resolved ion data is available from Galileo, but at energies of typically hundreds of $\mathrm{keV}$ per ion (e.g. Radioti et al., 2005), corresponding to the high-energy tail of the thermal populations of central interest here. The anisotropies of these energetic ions have been employed to study the flows in Jupiter's magnetosphere (e.g. Krupp et al., 2001; Woch et al., 2004), and have been used to identify flow bursts in the jovian tail attributed to mass-release and the Vasyliunas-cycle 
(Woch et al., 1999; Kronberg et al., 2005). At Saturn, hot plasma injections from the magnetotail have been observed by Cassini energetic neutral atom imaging data by Mitchell et al. (2005), and have been attributed to tail reconnection similar to substorms at Earth. These data include suprathermal fluxes of mass-resolved hydrogen and oxygen at energies from several tens to a few hundred $\mathrm{keV}$, again above the thermal ion energy range estimated here. Mass-resolved ion data at thermal energies should also become available from the Cassini plasma spectrometer (Young et al., 2004), but these observations may be difficult at the thermal plasma densities envisaged here (few times $0.01 \mathrm{~cm}^{-3}$ ). Nevertheless we hope that the theoretical discussion given here concerning the physical significance of such observations will provide impetus for detailed future studies of such data.

\section{Summary}

In this paper we have considered the contribution of the solar wind-driven Dungey-cycle to flux transport in the closed field regions of Jupiter's and Saturn's magnetospheres. Our estimates of the Dungey-cycle voltage in these systems have been derived from recent studies based on spacecraft measurements of the interplanetary medium near the orbits of these planets, combined with an empirical formula for the magnetopause reconnection rate validated at Earth. These values have then been compared with the voltages associated with the flows driven by planetary rotation. While the traditional comparison with the voltage associated with the rotational transport of the total planetary magnetic flux indicates that Dungey-cycle flows are negligible in these systems, being at most $\sim 1 \%$ of the rotational transport, here we point out that most of this rotational transport takes place in the innermost part of the system where the fields are strongest. If instead we consider only the rotational flows associated with the middle and outer regions of these magnetospheres, we find that while they are still more than an order of magnitude larger than the averaged Dungey-cycle voltages, and those occurring during solar wind rarefaction regions, they are of the same order as the typical Dungey-cycle voltages occurring in these systems during CIR compression regions and CMEs, namely $\sim 1 \mathrm{MV}$ at Jupiter and $\sim 150 \mathrm{kV}$ at Saturn. Under such conditions, therefore, the Dungey-cycle contributes a significant transport of magnetic flux within the outer parts of the closed field regions of these magnetospheres. Specifically, the width of the Dungey-cycle "return" flow layer inside the pre-noon dayside magnetopause is then estimated to be typically $\sim 4 R_{J}$ in Jupiter's magnetosphere and $\sim 2 R_{S}$ in Saturn's magnetosphere, occurring $\sim 10 \%$ of the time, compared with about one planetary radius or less during rarefaction regions. Thicker layers may occur if the tail reconnection is impulsive, but then lasting for correspondingly shorter intervals of time.
We also suggest that it may be possible to experimentally identify such layers inside these magnetospheres through the hot ion composition, provided the "blurring" effect of crossfield particle diffusion is not too strong. While regions driven by planetary rotation should be dominated by heavy-ion plasmas originating from internal moon sources, the Dungeycycle layers should principally contain hot light ions originating from either the planet's ionosphere or the solar wind. Estimates of the typical ion temperatures at Jupiter are $\sim 10 \mathrm{keV}$ in the tail and $\sim 20-30 \mathrm{keV}$ in the dayside outer magnetosphere, while for Saturn the energies are $\sim 2 \mathrm{keV}$ in the tail and $\sim 5 \mathrm{keV}$ ion the dayside outer magnetosphere.

Acknowledgements. Work at Leicester was supported by PPARC grant PPA/G/O/2003/00013. S. V. Badman was supported by a PPARC Quota Studentship, and S. W. H. Cowley by a Royal Society Leverhulme Trust Senior Research Fellowship.

Topical Editor I. A. Daglis thanks I. Dandouras and another referee for their help in evaluating this paper.

\section{References}

Acuña, M. H., Behannon, K. W., and Connerney, J. E. P.: Jupiter's magnetic field and magnetosphere, in: Physics of the Jovian Magnetosphere, edited by: Dessler, A. J., Cambridge Univ. Press, Cambridge, U.K., p. 1-50, 1983.

Arridge, C. S., Achilleos, N., Dougherty, M. K., Khurana, K. K., and Russell, C. T.: Modeling the size and shape of Saturn's magnetopause with variable dynamic pressure, J. Geophys. Res., 111, A11227, doi:10.1029/2005JA011574, 2006.

Badman, S. V., Bunce, E. J., Clarke, J. T., Cowley, S. W. H., Gérard, J.-C., Grodent, D., and Milan, S. E.: Open flux estimates in Saturn's magnetosphere during the January 2004 CassiniHST campaign, and implications for reconnection rates, J. Geophys. Res., 110, A11216, doi:10.1029/2005JA011240, 2005.

Badman, S. V., Cowley, S. W. H., Gérard, J.-C., and Grodent, D.: A statistical analysis of the location and width of Saturn's southern auroras, Ann. Geophys., 24, 3533-3545, 2006,

http://www.ann-geophys.net/24/3533/2006/.

Bagenal, F.: Empirical model of the Io plasma torus: Voyager measurements, J. Geophys. Res., 99, 11 043-11 062, 1994.

Behannon, K. W., Lepping, R. P., and Ness, N. F.: Structure and dynamics of Saturn's outer magnetosphere and boundary regions, J. Geophys. Res., 88, 8791-8800, 1983.

Bespalov, P. A., Davydenko, S. S., Cowley, S. W. H., and Nichols, J. D.: Interchange instability of the plasma disk in Jupiter's middle magnetosphere and its relation to the radial plasma density distribution, Ann. Geophys., 24, 2043-2055, 2006,

http://www.ann-geophys.net/24/2043/2006/.

Boudouridis, A., Zesta, E., Lyons, L. R., Anderson, P. C., and Lummerzheim, D.: Effect of solar wind pressure pulses on the size and strength of the auroral oval, J. Geophys. Res., 108, 80128027, doi:10.1029/2002JA009373, 2003.

Boudouridis, A., Zesta, E., Lyons, L. R., Anderson, P. C., and Lummerzheim, D.: Magnetospheric reconnection driven by solar wind pressure fronts, Ann. Geophys., 22, 1367-1378, 2004, http://www.ann-geophys.net/22/1367/2004/. 
Bunce, E. J. and Cowley, S. W. H.: A note on the ring current in Saturn's magnetosphere: Comparison of magnetic data obtained during the Pioneer-11 and Voyager-1 and -2 fly-bys, Ann. Geophys., 21, 661-669, 2003, http://www.ann-geophys.net/21/661/2003/.

Bunce, E. J., Cowley, S. W. H., Wright, D. M., Coates, A. J., Dougherty, M. K., Krupp, N., Kurth, W. S., and Rymer, A. M.: In-situ observations of a solar wind compression-induced hot plasma injection in Saturn's tail, Geophys. Res. Lett., 32, L20S04, doi:10.1029/2005GL022888, 2005.

Clarke, J. T., Gérard, J.-C., Grodent, D., Wannawichian, S., Gustin, J., Connerney, J., Crary, F., Dougherty, M., Kurth, W., Cowley, S. W. H., Bunce, E. J., Hill, T., and Kim, J.: Morphological differences between Saturn's ultraviolet aurorae and those of Earth and Jupiter, Nature, 433, 717-719, 2005.

Connerney, J. E. P., Acuna, M. H., Ness, N. F.: Currents in Saturn's magnetosphere, J. Geophys. Res., 88, 8779-8789, 1983.

Connerney, J. E. P., Acuña, M. H., Ness, N. F., and Satoh, T.: New models of Jupiter's magnetic field constrained by the Io flux tube footprint, J. Geophys. Res., 103, 11 929-11940, 1998.

Cowley, S. W. H.: The distant geomagnetic tail in theory and observation, in: Magnetic Reconnection in Space and Laboratory Plasmas, edited by: Hones Jr., E.W., American Geophysical Union, Washington, D.C., USA, p. 228-239, 1984.

Cowley, S. W. H. and Bunce, E. J.: Corotation-driven magnetosphere-ionosphere coupling currents in Saturn's magnetosphere and their relation to the auroras, Ann. Geophys., 21, 1691-1707, 2003,

http://www.ann-geophys.net/21/1691/2003/.

Cowley, S. W. H., Balogh, A., Dougherty, M. K., Dunlop, M. W., Edwards, T. M., Forsyth, R. J., Laxton, N. F., and Staines, K.: Plasma flow in the jovian magnetosphere and related magnetic effects: Ulysses observations, J. Geophys. Res., 101, 15 197$15210,1996$.

Cowley, S. W. H., Nichols, J. D., and Bunce, E. J.: Distributions of current and auroral precipitation in Jupiter's middle magnetosphere computed from steady-state Hill-Pontius angular velocity profiles: Solutions for current sheet and dipole magnetic field models, Planet. Space Sci., 50, 717-734, 2002.

Cowley, S. W. H., Davies, J. A., Grocott, A., Khan, H., Lester, M., McWilliams, K. A., Milan, S. E., Provan, G., Sandholt, P. E., Wild, J. A., and Yeoman, T. K.: Solar wind-magnetosphereionosphere interactions in the Earth's plasma environment, Phil. Trans. A, 361, 113-126, 2003a.

Cowley, S. W. H., Bunce, E. J., Stallard, T. S., and Miller, S.: Jupiter's polar ionospheric flows: theoretical interpretation, Geophys. Res. Lett., 30, 1220-1223, doi:10.1029/2002GL016030, 2003 b.

Cowley, S. W. H., Bunce, E. J., and Prangé, R.: Saturn's polar ionospheric flows and their relation to the main auroral oval, Ann. Geophys., 22, 1379-1394, 2004a.

Cowley, S. W. H., Bunce, E. J., and O'Rourke, J. M.: A simple quantitative model of plasma flows and currents in Saturn's polar ionosphere, J. Geophys. Res., 109, A05212, doi:10.1029/2003JA010375, 2004b.

Cowley, S. W. H., Badman, S. V., Bunce, E. J., Clarke, J. T., Gérard, J.-C., Grodent, D., Jackman, C. M., Milan, S. E., and Yeoman, T. K.: Reconnection in a rotation-dominated magnetosphere and its relation to Saturn's auroral dynamics, J. Geo- phys. Res., 110, A02201, doi:10.1029/2004JA010796, 2005a.

Cowley, S. W. H., Alexeev, I. I., Belenkaya, E. S., Bunce, E. J., Cottis, C. E., Kalegaev, V. V., Nichols, J. D., Prangé, R., and Wilson, F. J.: A simple axi-symmetric model of magnetosphereionosphere coupling currents in Jupiter's polar ionosphere, J. Geophys. Res., 110, A11209, doi:10.1029/2005JA011237, 2005 b.

Dougherty, M. K., Achilleos, N., Andre, N., et al.: Cassini magnetometer observations during Saturn orbit insertion, Science, 307, 1266-1270, 2005.

Dungey, J. W.: Interplanetary field and the auroral zones, Phys. Rev. Lett., 6, 47-48, 1961.

Frank, L. A., Paterson, W. R., and Khuarana, K. K.: Observations of thermal plasmas in Jupiter's magnetotail, J. Geophys. Res., 107, A11003, doi:10.1029/2001JA000077, 2002.

Frank, W. A. and Paterson, W. R.: Plasmas observed near local noon in Jupiter's magnetosphere with the Galileo spacecraft, J. Geophys. Res., 109, A11217, doi:10.1029/2002JA009795, 2004.

Gérard, J.-C., Grodent, D., Gustin, J., Saglam, A., Clarke, J. T., and Trauger, J. T.: Characteristics of Saturn's FUV aurora observed with the Space Telescope Imaging Spectrograph, J. Geophys. Res., 109, A09207, doi:10.1029/2004JA010513, 2004.

Grodent, D., Clarke, J. T., Kim, J., Waite Jr., J. H., and Cowley, S. W. H.: Jupiter's main oval observed with HST-STIS, J. Geophys. Res., 108, 1389-1404, doi:10.1029/2003JA009921, 2003a.

Grodent, D., Clarke, J. T., Waite Jr., J. H., Cowley, S. W. H., Gérard, J.-C., and Kim, J.: Jupiter's polar auroral emissions, J. Geophys. Res., 108, 1366-1374, doi:10.1029/2003JA010017, 2003b.

Grodent, D., Gérard, J.-C., Cowley, S. W. H., Bunce, E. J., and Clarke, J. T.: Variable morphology of Saturn's southern ultraviolet aurora, J. Geophys. Res., 110, A07215, doi:10.1029/2004JA010983, 2005.

Gurnett, D. A., Kurth, W. S., Hospodarsky, G. B., et al.: Radio and plasma wave observations at Saturn from Cassini's approach and first orbit, Science, 307, 1255-1259, 2005.

Gustin, J., Gérard, J.-C., Grodent, D., Cowley, S. W. H., Clarke, J. T., and Grard, A.: Energy-flux relationship in the FUV jovian aurora deduced from HST-STIS spectral observations, J. Geophys. Res., 109, A10205, doi:10.1029/2003JA010365, 2004.

Gustin, J., Cowley, S. W. H., Gérard, J.-C., Gladstone, G. R., Grodent, D., and Clarke, J. T.: Characteristics of jovian morning bright FUV aurora from HST/STIS imaging and spectral observations, J. Geophys. Res., 111, A09220, doi:10.1029/2006JA011730, 2006.

Hartle, R. E., Sittler Jr., E. C., Neubauer, F. M., Johnson, R. E., Smith, H. T., Crary, F., McComas, D. J., Young, D. T., Coates, A. J., Simpson, D., Bolton, S., Reisenfeld, D., Szego, K., Berthelier, J. J., Rymer, A., Vilppola, J., Steinberg, J. T., and André, N.: Preliminary interpretation of Titan plasma interaction as observed by the Cassini Plasma Spectrometer: Comparisons with Voyager 1, Geophys. Res. Lett., 33, L08201, doi:10.1029/2005GL024817, 2006.

Hill, T. W.: Inertial limit on corotation, J. Geophys. Res., 84, 6554 6558, 1979.

Hill, T. W., Dessler, A. J., and Goertz, C. K.: Magnetospheric models, in: Physics of the Jovian Magnetosphere, edited by: Dessler, A. J., Cambridge Univ. Press, Cambridge, UK, p. 353 394, 1983. 
Jackman, C. M., Achilleos, N., Bunce, E. J., Cowley, S. W. H., Dougherty, M. K., Jones, G. H., Milan, S. E., and Smith, E. J.: Interplanetary magnetic field at $\sim 9 \mathrm{AU}$ during the declining phase of the solar cycle and its implications for Saturn's magnetospheric dynamics, J. Geophys. Res., 109, A11203, doi:10.1029/2004JA010614, 2004.

Jackman, C. M. and Cowley, S. W. H.: A model of the plasma flow and current in Saturn's polar ionosphere under conditions of strong Dungey-cycle driving, Ann. Geophys., 24, 1029-1055, 2006, http://www.ann-geophys.net/24/1029/2006/.

Joy, S. P., Kivelson, M. G., Walker, R. J., Khurana, K. K., Russell, C. T., and Ogino, T.: Probabilistic models of the jovian magnetopause and bow shock locations, J. Geophys. Res., 107, 1309-1325, doi:10.1029/2001JA009146, 2002.

Kane, M., Mauk, B. H., Keath, E. P., and Krimigis, S. M.: Hot ions in the jovian magnetodisc: A model for Voyager 2 low-energy charged particle measurements, J. Geophys. Res., 100, 1947319486, 1995.

Kennel, C. F. and Coroniti, F. V.: Is Jupiter's magnetosphere like a pulsar's or Earth's?, in: The magnetospheres of Earth and Jupiter, edited by: Formisano, V., D. Reidel, Norwell, Mass., p. 451-477, 1975.

Khurana, K. K. and Kivelson, M. G.: Inference of the angular velocity of plasma in the jovian magnetosphere from the sweepback of magnetic field, J. Geophys. Res., 98, 67-80, 1993.

Kivelson, M. G. and Southwood, D. J.: Dynamical consequences of two modes of centrifugal instability in Jupiter's outer magnetosphere, J. Geophys. Res., 110, A12209, doi:10.1029/2003JA011176, 2005.

Krimigis, S. M., Mitchell, D. G., Hamilton, D. C., et al.: Dynamics of Saturn's magnetosphere from MIMI during Cassini's orbital insertion, Science, 307, 1270-1273, 2005.

Kronberg, E. A., Woch, J., Krupp, N., Lagg, A., Khurana, K. K., and Glassmeier, K.-H.: Mass release at Jupiter: Substorm-like processes in the jovian magnetotail, J. Geophys. Res., 110, A03211, doi:10.1029/2004JA010777, 2005.

Krupp, N., Lagg, A., Livi, S., Wilken, B., Woch, J., Roelof, E. C., and Williams, D. J.: Global flows of energetic ions in Jupiter's equatorial plane: First-order approximation, J. Geophys. Res., 106, 26 017-26 032, 2001.

Louarn, P., Roux, A., Perraut, S., Kurth, W. S., and Gurnett, D. A.: A study of the jovian "energetic magnetospheric events" observed by Galileo: role in the radial plasma transport, J. Geophys. Res., 105, 13 073-13 088, 2000.

Lyons, L. R., Blanchard, G. T., Samson, J. C., Lepping, R. P., Yamamoto, T., and Moretto, T.: Coordinated observations demonstrating external substorm triggering, J. Geophys. Res, 102, 27 039-27 051, 1997.

Milan, S. E., Cowley, S. W. H., Lester, M., Wright, D. M., Slavin, J. A., Fillingim, M., Carlson, C. W., and Singer, H. J.: Response of the magnetotail to changes in the open flux content of the magnetosphere, J. Geophys. Res., 109, A04220, doi:10.1029/2003JA010350, 2004.

Milan, S. E., Provan, G., and Hubert, B.: Magnetic flux transport in the Dungey cycle: A survey of dayside and nightside reconnection rates, J. Geophys. Res., 112, A01209, doi:10.1029/2006JA011642, 2006.

Mitchell, D. G., Brandt, P. C., Roelof, E. C., Dandouras, J., Krim- igis, S. M., Mauk, B. M., Paranicas, C. P., Krupp, N., Hamilton, D. C., Kurth, W. S., Zarka, P., Dougherty, M. K., Bunce, E. J., Shemansky, D. E.: Energetic ion acceleration in Saturn's magnetotail: Substorms at Saturn?, Geophys. Res. Lett., 32, L20S01, doi:10.1029/2005GL022647, 2005.

Ness, N. F., Acuña, M. H., Lepping, R. P., Connerney, J. E. P., Behannon, K. W., Burlaga, L. F., and Neubauer, F. M.: Magnetic field studies by Voyager 1: Preliminary results at Saturn, Science, 212, 211-217, 1981.

Nichols, J. D. and Cowley, S. W. H.: Magnetosphere-ionosphere coupling currents in Jupiter's middle magnetosphere: effect of precipitation-induced enhancement of the ionospheric Pedersen conductivity, Ann. Geophys., 22, 1799-1827, 2004, http://www.ann-geophys.net/22/1799/2004/.

Nichols, J. D. and Cowley, S. W. H.: Magnetosphere-ionosphere coupling currents in Jupiter's middle magnetosphere: effect of magnetosphere-ionosphere decoupling by field-aligned auroral voltages, Ann. Geophys., 23, 799-808, 2005, http://www.ann-geophys.net/23/799/2005/.

Nichols, J. D., Cowley, S. W. H., and McComas, D. J.: Magnetopause reconnection rate estimates for Jupiter's magnetosphere based on interplanetary measurements at $\sim 5 \mathrm{AU}$, Ann. Geophys., 24, 393-406, 2006,

http://www.ann-geophys.net/24/393/2006/.

Nichols, J. D., Bunce, E. J., Clarke, J. T., Cowley, S. W. H., Gérard, J.-C., Grodent, D., and Pryor, W. R.: Response of Jupiter's UV auroras to interplanetary conditions as observed by the Hubble Space Telescope during the Cassini fly-by campaign, J. Geophys. Res., 112, A02203, doi:10.1029/2006JA012005, 2007.

Paranicas, C., Mitchell, D. G., Roelof, E. C., Brandt, P. C., Williams, D. J., Krimigis, S. M., and Mauk, B. H.: Periodic intensity variations in global ENA images of Saturn, Geophys. Res. Lett., 32, L21101, doi:10.1029/2005GL023656, 2005.

Perrault, P. and Akasofu, S.-I.: A study of geomagnetic storms, Geophys. J. Roy. Astron. Soc., 54, 547-573, 1978.

Pontius Jr., D. H.: Radial mass transport and rotational dynamics, J. Geophys. Res., 102, 7137-7150, 1997.

Radioti, A., Krupp, N., Woch, J., Lagg, A., Glassmeier, K.-H., and Waldrop, L. S.: Ion abundance ratios in the Jovian magnetosphere, J. Geophys. Res., 110, A07225, doi:10.1029/2004JA010775, 2005.

Richardson, J. D.: Thermal ions at Saturn: Plasma parameters and implications, J. Geophys. Res., 91, 1381-1389, 1986.

Richardson, J. D. and Sittler Jr., E.C.: A plasma density model for Saturn based on Voyager observations, J. Geophys. Res., 95, 12 019-12 031, 1990.

Russell, C. T., Khurana, K. K., Huddleston, D. E., Kivelson, M. G.: Localized reconnection in the near jovian magnetotail, Science, 280, 1061-1064, 1998.

Siscoe, G. L. and Summers, D.: Centrifugally-driven diffusion of iogenic plasma, J. Geophys. Res., 86, 8471-8479, 1981.

Southwood, D. J. and Kivelson, M. G.: Magnetospheric interchange motion, J. Geophys. Res., 94, 299-308, 1989.

Szego, K., Bebesi, Z., Erdos, G., Foldy, L., Crary, F., McComas, D. J., Young, D. T., Bolton, S., Coates, A. J., Rymer, A. M., Hartle, R. E., Sittler, E. C., Reisenfeld, D., Bethelier, J. J., Johnson, R. E., Smith, H. T., Hill, T. W., Vilppola, J., Steinberg, J., and Andre, N..: The global plasma environment of Titan as 
observed by Cassini Plasma Spectrometer during the first two close encounters with Titan, Geophys. Res. Lett., 32, L20S05, doi:10.1029/2005GL022646, 2005.

Vasyliunas, V. M.: Plasma distribution and flow, in: Physics of the Jovian Magnetosphere, edited by: Dessler, A. J., Cambridge Univ. Press, Cambridge, UK, p. 395-453, 1983.

Waldrop, L. S., Fritz, T. A., Kivelson, M. G., Khurana, K., Krupp, N., and Lagg, A.: Jovian plasma sheet morphology: Particle and field observations by the Galileo spacecraft, Planet. Space Sci., 53, 681-692, 2005.

Woch, J., Krupp, N., Khurana, K. K. Kivelson, M. G., Roux, A., Perraut, S., Louarn, P., Lagg, A., Williams, D. J., Livi, S., and Wilken, B.: Plasma sheet dynamics in the Jovian magnetotail: Signatures for substorm-like processes?, Geophys. Res. Lett., 26, 2137-2140, 1999.
Woch, J., Krupp, N., Lagg, A., and Tomás, A.: The structure and dynamics of the jovian energeric particle distribution, Adv. Space. Res., 33, 2030-2038, 2004.

Young, D. T., Berthelier, J.-J., Blanc, M., et al.: The Cassini plasma spectrometer investigation, Space Sci. Rev., 114, 1-112, 2004.

Young, D. T., Berthelier, J.-J., Blanc, M., et al.: Composition and dynamics of plasma in Saturn's magnetosphere, Science, 307, 1262-1266, 2005. 